\title{
Low platelet to lymphocyte ratio and high platelet distribution width have an inferior outcome in chronic lymphocytic leukaemia patients
}

\author{
Shaimaa El-Ashwah' ${ }^{1}$, May Denewer ${ }^{1}$, Nermeen A. Niazy², \\ Metwaly I. Mortada ${ }^{3}$, Emad Azmy ${ }^{1}$
${ }^{1}$ Clinical Hematology Unit, internal Medicine Department, Oncology Center, Mansoura University, Mansoura, Egypt 2Public Health and Community Medicine, Faculty of Medicine, Mansoura University, Mansoura, Egypt ${ }^{3}$ Hematology Unit, Clinical Pathology Department, Faculty of Medicine, Mansoura University, Mansoura, Egypt

\begin{abstract}
Introduction. Chronic lymphocytic leukaemia (CLL) is an incurable disease of the elderly, characterised by gradual accumulation of small mature B lymphocytes which escape apoptosis through inflammatory signals from the microenvironment. Elevated inflammatory markers are associated with very poor prognosis in different types of cancer. Therefore, we examined retrospectively the impact of platelet lymphocyte ratio (PLR) and platelet distribution width (PDW) on 180 CLL patients' outcome.
\end{abstract}

Materials and methods. This retrospective study included 180 patients with CLL who were diagnosed and selected among cases referred to the Oncology Center Mansoura University between January $1^{\text {st }}, 2008$ and June $30^{\text {th }}, 2016$. All the relevant information was collected from the electronic medical records of the selected patients.

Results. Our results revealed that low PLR $(<2.5)$ was more frequently observed in patients with stage $C(p<0.001)$, with 17p deletion ( $p=0.017$ ), and CD38 expression $(p=0.08)$, but not with seropositive HCV patients $(p=0.2)$. High PDW ( $\geq 18.5$ fl) was more frequently associated with intention to treat population ( $p=0.038)$, and CD38 expression $(p=0.068)$, but not with $17 p$ deletion $(p=0.25$ ) and seropositive HCV patients $(p=0.4)$. Multivariate analysis for overall survival showed that stage $A$ and low PDW were independent factors for overall survival ( $p=0.014$ and 0.04 respectively), while high PLR ( $p=$ $0.05)$, and seronegative HCV patients $(p=0.1)$ lost their significance.

Conclusion. Our data showed that low PLR and high PDW were associated with poor prognostic markers. Stage C-CLL and high PDW were independent predictors of survival.

Key words: chronic lymphocytic leukaemia, platelet distribution width, platelet-to-lymphocyte ratio

\section{Introduction}

Chronic lymphocytic leukaemia (CLL) is an incurable disease that is characterized by gradual accumulation of small mature B lymphocytes [1]. These lymphocytes are dormant replicational cells that accumulate in the marrow and peripheral blood, due to extrinsic survival signals from the microenvironment [2].
These leukaemic lymphocytes can resist apoptosis by inflammatory signals compared to normal B lymphocytes. Actually, CLL patients present with manifestations that typically occur in chronic inflammatory disorders which make the role of inflammation clear [3]. Thrombocytopenia in CLL patients caused by either bone marrow infiltration, immune thrombocytopenia, hypersplenism, or myelosuppression secondary to cytotoxic

\section{How to cite:}

El-Ashwah S, Denewer M, Niazy NA, Mortada MI, Azmy E. Low platelet to lymphocyte ratio and high platelet distribution width have an inferior outcome in chronic lymphocytic leukaemia patients. NOWOTWORY J Oncol 2020; 70: 121-126. 
therapy or infection [4]. PLR is a novel inflammatory marker that can be applied in many diseases for predicting inflammation, and PDW represents the platelet anisocytosis and is calculated from the distribution of individual platelet volumes [5].

Further, platelet-to-lymphocyte ratio (PLR) finds its role in CLL that the lymphocyte and platelet counts are correlated to the pathogenesis of CLL directly and affect management of patients. Also, PDW does not assess heterogeneity of platelet volume only, but also platelet activity [6]. Many studies have shown that these two inflammatory biomarkers (PLR and PDW) are considered prognostic factors for some non-haematological tumours [5].

To our knowledge, PDW has not been studied in CLL. So, in our study, we aimed at investigating the role of PLR and PDW in our CLL patients.

\section{Materials and methods}

\section{Subjects}

This retrospective study included 180 patients with CLL who were diagnosed and selected among cases referred to the Oncology Center Mansoura University (OCMU) between January $1^{\text {th }}, 2008$ and June $30^{\text {th }}, 2016$. All the relevant information was obtained through the electronic medical records of the selected patients. All laboratory procedures were performed in the clinical pathology labs of OCMU. The Binet staging system was used to classify the CLL patients:

- Binet stage A: $<3$ areas of lymphoid tissue are enlarged, with no anaemia or thrombocytopenia.

- Binet stage B: $\geq 3$ areas of lymphoid tissue are enlarged, with no anaemia or thrombocytopenia.

- Binet stage C: anaemia ( $<10 \mathrm{~g} / \mathrm{dL}$ ) and/or thrombocytopenia $\left(<100 \times 10^{9} / \mathrm{L}\right)$ are present. Any number of lymphoid tissue areas may be enlarged.

They were treated according to our institute guidelines based on performance statue by purine based regimen or alkylators. As far as we know, patients with immune-related cytopenia or infection were excluded from our study.

\section{Patient evaluation}

Detailed history taking and clinical examination. Laboratory investigations:

1. Routine work:

- Complete blood count (haemogram): using the electronic counter (CELL-DYN 3700, Abbott, Canada), PDW and PLR were obtained, before any treatment, including PDW (fl), the lymphocyte count (k/uL) and platelet count (k/uL). We calculated the PLR by dividing the absolute count of platelets to that of lymphocytes at diagnosis with thorough examination of peripheral blood smears stained with Leishman stain.

- Liver function tests, serum creatinine, serum uric acid, and serum LDH.

- Virology screen (HCV, HBsAg, HIV): HCV Ab was detected using Murex HCV Ag/Ab Combination $4^{\text {th }}$ generation ELISA kit \# 4J2453 Anti-Core monoclonal antibody, recombinant antigen and peptides representing the immunodominant regions of NS3 and core. Simultaneously, the Bioelisa ELISA kit was used for detection of Hepatitis B surface antigen (HBsAg). Genscreen ${ }^{\text {TM }}$ ULTRA HIV Ag-Ab. The Genscreen ${ }^{\text {TM }}$ ULTRA HIV Ag-Ab is a qualitative enzyme immunoassay kit for the detection of HIV p24 antigen and antibodies to HIV-1 (groups M and O) and HIV-2 in human serum or plasma.

2. Work up for CLL diagnosis:

- Microscopic study of bone marrow and peripheral smears.

- Immunophenotyping (IPT) using American (BD FACSCANTOII) to diagnose the cases and exclude other types of lymphoma by incubation of washed cells from peripheral blood or bone marrow samples with fluorescein-labelled monoclonal antibody including scoring system of CLL (CD5, CD19,CD23,CD79b,slgM,CD38), kappa and lambda. Positivity in each marker can be calculated if it is more than $20 \%$.

- FISH for detection of 17p deletion. Interphase FISH technique was conducted on peripheral blood or bone marrow aspiration and trephine. Using the Olympus BX 61, fluorescent microscope. Interphase FISH technique was performed on samples after optimization of the protocol using commercially available probe from Cytocell UK LPH TP53 deletion FISH Probe Kit.

\section{Statistical analysis}

Data were analysed on a personal computer running SPSSC for Windows (Statistical Package for Social Scientists) Release 18. A two-tailed $p$ value of $>0.05$ was considered statistically significant. For descriptive statistics of qualitative variables, the frequency distribution procedure was run with calculation of the number of cases and percentages. For descriptive statistics of quantitative variables, the median and range were used. Association between categorical variables was tested by the Chi Square Test or Fishers exact test. The independent-samples t-test was used to compare the means between two groups. Time to treat was defined as the time from diagnosis until the start of chemotherapy or death. Overall survival was calculated by the Kaplan-Meier Product-Limit Estimator. Comparison of the survival was performed by the Log-Rank Test. Exploring variables for their independent prognostic effect on survival was carried out using the multivariate stepwise Cox's proportional regression hazard model.

\section{Results}

The 180 CLL patients were (101 M; 79 F) with mean age $60.27 \pm 11.49$ years. The incidence of chronic HCV infection and HBV in our study were $38.3 \%$ and $3.9 \%$ respectively. At diagnosis, the median PLR was 2.5 (range 0.07-42), platelets $138.5 \mathrm{k} / \mu \mathrm{L}$ (range $5-459 \mathrm{k} / \mu \mathrm{L}$ ), and the median PDW was 18.5 (range 15.6-24.9). Basic data are illustrated in table I. 
Table I. Basic data of studied cases

\begin{tabular}{|c|c|c|}
\hline Character & Value & Percentage \\
\hline Male/female & $101 / 79$ & $56.1 \% / 43.9 \%$ \\
\hline HCV positive & 69 & $38.3 \%$ \\
\hline HBV positive & 7 & $3.9 \%$ \\
\hline \multirow[t]{2}{*}{ B symptoms - present } & 107 & $59 \%$ \\
\hline & Median & Range \\
\hline WBC (k/uL) & 61.85 & $8-960$ \\
\hline $\mathrm{ALC}(\mathrm{k} / \mathrm{uL})$ & 52.5 & $6-900$ \\
\hline $\mathrm{HB}(\mathrm{g} / \mathrm{dl})$ & 11 & $4.3-16.2$ \\
\hline Platelet (k/uL) & 138.5 & $5-459$ \\
\hline PLR & 2.5 & $0.07-42$ \\
\hline PDW & 18.5 & $15.6-24.9$ \\
\hline Stage & Number & $\%$ \\
\hline A & 3 & $1.7 \%$ \\
\hline B & 94 & $52.2 \%$ \\
\hline C & 83 & $46.1 \%$ \\
\hline \multicolumn{3}{|l|}{ Prognostic markers } \\
\hline 17p deletion positive $(n=35)$ & 5 & $14.28 \%$ \\
\hline CD38 positive $(n=93)$ & 33 & $35.48 \%$ \\
\hline ZAP-70 positive $(n=30)$ & 18 & $60 \%$ \\
\hline PLR & Value & No (\%) \\
\hline Low PLR & $<2.5$ & $86(47.8 \%)$ \\
\hline High PLR & $\geq 2.5$ & $94(52.2 \%)$ \\
\hline PDW & & No (\%) \\
\hline Low PDW & $<18.5 \mathrm{fl}$ & $82(45.6 \%)$ \\
\hline High PDW & $\geq 18.5 f$ & $98(54.4 \%)$ \\
\hline Intention to treat - population & 138 & $76.7 \%$ \\
\hline Treatment protocol & No & $\%$ \\
\hline Wait and watch & 19 & $10.6 \%$ \\
\hline Purine based & 53 & $29.4 \%$ \\
\hline Alkylators based & 108 & $60 \%$ \\
\hline Status (alive/dead) & $115 / 65$ & $63.9 \% / 36.1 \%$ \\
\hline
\end{tabular}

Low PLR $(<2.5)$ was more frequently observed in male patients $(p=0.06)$ with stage $C(p<0.001)$, with 17p deletion $(p=0.017)$, and CD38 expression $(p=0.08)$ and intention to treat $(p<0.001)$, but not with HCV seropositive patients $(p=0.22)$ and ZAP-70 positivity $(p=0.28)$ (table II).

High PDW ( $\geq 18.5 \mathrm{fl}$ ) was more frequently associated with intention to treat population ( $p=0.038$ ), and CD38 expression $(p=0.068)$, but not with $17 p$ deletion $(p=0.25)$ and seropositive HCV patients $(p=0.43$ ) (table III).

The median time to initiate treatment in CLL patients was 2.05 years. It was found that the majority of intention to treat population was associated with low PLR ( $<0.001)$, high PDW ( $p=0.038)$, seropositive HCV ( $p 0.027)$ and seropostive HBV $(p=0.2)$.

The median overall survival of the studied group was 5.58 years. CLL patients with stage $A$, hepatitis $C$ seronegative patients, low PDW, high PLR were associated with superior overall survival with significant value $(p=0.001,0.017,0.043$, and 0.002 respectively figure $1 \mathrm{a}, \mathrm{b}, \mathrm{c}$ ). Multivariate analysis showed that stage A and low PDW were independent factors for OS ( $p=0.014$ and 0.04 respectively), while high PLR $(p=0.05)$, and seronegative $C(p=0.1)$ lost their significance.

\section{Discussion}

CLL is considered a heterogeneous disorder associated with different clinical courses which were predicted by staging systems of Binet and Rai. However, these systems do not consider other CLL biological features which can affect the course of the disease $[7,8]$.

Hitherto, new molecular advances have resulted in the use of expensive and complicated prognostic markers like cytogenetic aberrations (17p deletions, $11 q$ deletions and trisomy 12), $\beta 2$ micro-globulin, IGHV mutational status, expression of CD38 and ZAP-70 and gene mutations like NOTCH1, MYD88 and SF3B1 [9].

Unfortunately, most of these biomarkers were not taken in all of our cases because of either the cost and or unavailability. Another limitation to this study would be immune thrombocytopenia. However, to the best of our knowledge,

Table Il. Comparison between low PLR and high PLR group in CLL patients

\begin{tabular}{|c|c|c|c|c|c|}
\hline & & Low PLR (<2.5) & High PLR ( $\geq 2.5$ ) & Test of significance & p \\
\hline \multicolumn{2}{|l|}{ Male } & $59(62.76 \%)$ & $42(48.84 \%)$ & 3.53 & 0.06 \\
\hline \multicolumn{2}{|c|}{ Age $>65$} & $34(36.17 \%)$ & $29(33.72 \%)$ & 0.12 & 0.7 \\
\hline \multicolumn{2}{|c|}{ HCV positive } & $40(42.55 \%)$ & $29(33.72 \%)$ & 1.48 & 0.22 \\
\hline \multicolumn{2}{|c|}{ HBV positive } & $4(4.26 \%)$ & $3(3.48 \%)$ & 0.07 & 0.54 \\
\hline \multirow[t]{3}{*}{ Stage } & A & $1(1.06 \%)$ & $2(2.33 \%)$ & \multirow{3}{*}{19.26} & \multirow{3}{*}{$<0.001$} \\
\hline & B & $35(37.23 \%)$ & $59(68.6 \%)$ & & \\
\hline & C & $58(58 \%)$ & $25(29.06 \%)$ & & \\
\hline \multicolumn{2}{|c|}{ Intention to treat population } & $83(88.29 \%)$ & $55(63.95 \%)$ & 14.87 & $<0.001$ \\
\hline \multicolumn{2}{|c|}{ CD38 positive } & $21(43.75 \%)$ & $12(26.66 \%)$ & 2.96 & 0.08 \\
\hline \multicolumn{2}{|c|}{ ZAP-70 positive } & $10(71.4 \%)$ & $8(50 \%)$ & 1.4 & 0.28 \\
\hline \multicolumn{2}{|c|}{ del (17p) } & $5(23.8 \%)$ & $0(0 \%)$ & 5.65 & 0.017 \\
\hline
\end{tabular}


Table III. Comparison between low PDW and high PDW group in CLL patients

\begin{tabular}{|c|c|c|c|c|c|}
\hline & Low PDW (<18.5 fl) & High PDW ( $\geq 18.5$ fl) & Test of significance & $\mathrm{p}$ \\
\hline \multicolumn{2}{|l|}{ Male } & $42(51.2 \%)$ & $59(60.2 \%)$ & 1.46 & 0.23 \\
\hline \multicolumn{2}{|c|}{ Age $>65$} & $24(29.3 \%)$ & $39(39.8 \%)$ & 2.17 & 0.14 \\
\hline \multicolumn{2}{|c|}{ HCV positive } & 34 (41.5\%) & 35 (35.7\%) & 0.62 & 0.43 \\
\hline \multicolumn{2}{|c|}{ HBV positive } & $4(4.9 \%)$ & $3(3.1 \%)$ & 0.39 & 0.53 \\
\hline \multirow[t]{3}{*}{ Stage } & A & $2(2.4 \%)$ & $1(1 \%)$ & \multirow{3}{*}{1.12} & \multirow{3}{*}{0.56} \\
\hline & B & $45(54.9 \%)$ & $49(50 \%)$ & & \\
\hline & C & $35(42.7 \%)$ & $48(49 \%)$ & & \\
\hline \multicolumn{2}{|c|}{ Intention to treat population } & $57(69.5 \%)$ & $81(82.7 \%)$ & 4.31 & 0.038 \\
\hline \multicolumn{2}{|c|}{ CD38 positive } & $9(24.3 \%)$ & $24(42.9 \%)$ & 3.34 & 0.068 \\
\hline \multicolumn{2}{|c|}{ ZAP-70 positive } & $7(43.8 \%)$ & $11(78.6 \%)$ & 3.77 & 0.052 \\
\hline \multicolumn{2}{|c|}{ del (17p) } & $2(28.6 \%)$ & $3(10.7 \%)$ & 1.45 & 0.227 \\
\hline
\end{tabular}

Survival Functions

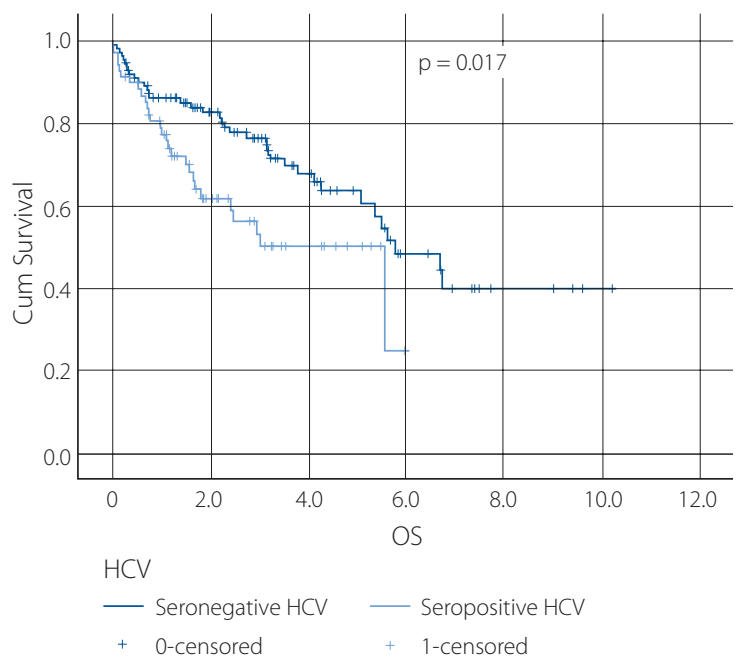

Figure 1a. Effect of HCV infection on overall survival of studied population

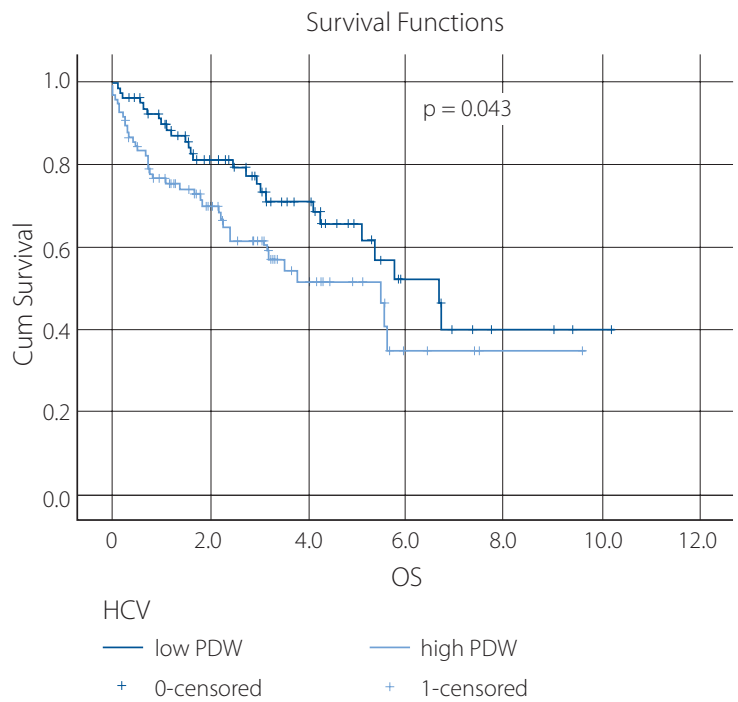

Figure 1 $\mathbf{b}$. Effect of PDW on overall survival of studied population

patients with auto-immune hematologic manifestations were not included in this analysis. Only two patients presented

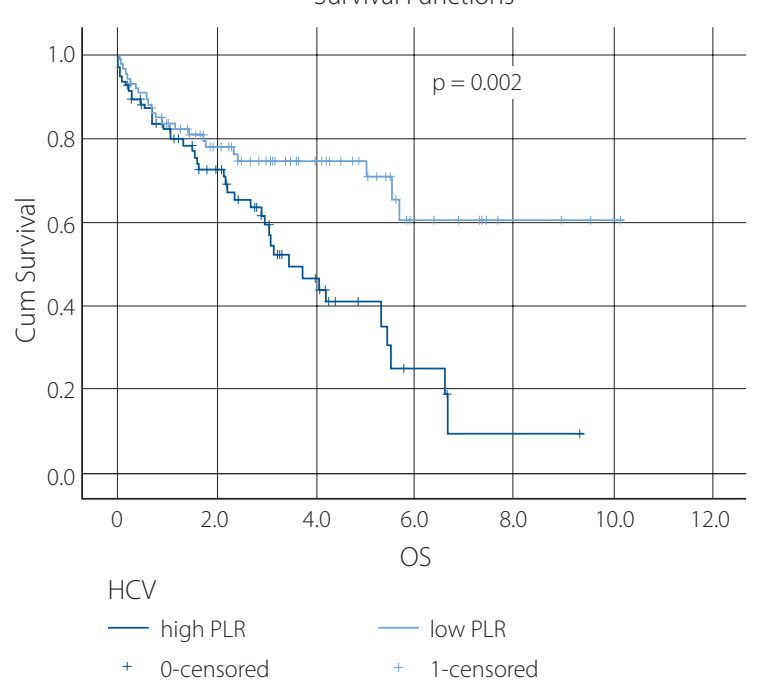

Figure 1c. Effect of PLR on overall survival of studied population

extreme thrombocytopenia and their work up did not reveal an immune phenomenon.

Recently, correlation between cancer and inflammation is an important new area of research. The antitumour activity of inflammation and the associated immune activation, induce tumour growth and progression. Inflammation is an independent predictor for response to therapy, event-free survival and overall survival (OS) in diffuse large B-cell lymphoma (DLBCL) patients [10].

Molica et al. have reported that in newly diagnosed CLL patients, the doubling time of absolute lymphocytic count was an independent predictor of outcomes in those patients [11]. Although platelet count prognostic value in CLL is not well identified, thrombocytopenia is considered a treatment indication [6]. Also, some studies have found that thrombocytopenia results in a compensatory thrombopoietin release which might correlate to some prognostic markers like ZAP-70 and CD38 $[12,13]$. So, we used the PLR as it is an easily applicable clinical method that could detect the patients with a poor prognosis early. 
Cytopenia in patients with CLL can have multiple causes including progressive bone marrow (BM) infiltration by abnormal lymphocytes, autoimmune disease, therapy-related, non-CLL related disorders, or a combination of these mechanisms [14]. The biological rationale in calculating PLR is that lymphocytosis and thrombocytopenia often occured in the advanced stages of CLL [6].

Our data demonstrated that Low PLR $(<2.5)$ group was significantly associated with poor prognostic markers; stage $C(p<0.001)$, with 17p deletion $(p=0.017)$, and intention to treat $(p<0.001)$. They had significantly shorter OS compared to high PLR ( $p=0.002)$ in a univariate analysis, while they lost their significance in multivariate analysis ( $p=0.05$ ). In solid tumours, a positive relationship between high PLR with worse prognosis for colorectal, gastroesophageal, hepatocellular, pancreatic, and ovarian cancers was identified [15].

Meanwhile, Kang et al. demonstrated that PLR had significant association with a poor prognosis in patients with non-Hodgkin's lymphoma, treated by R-CHOP [16]. Wang et al. reported that high PLR was associated with shorter OS and PFS in patients with DLBCL [10], also Seo et al. found that PLR showed independent significance in patients with advanced stage marginal zone lymphoma treated with rituximab, vincristine, cyclophosphamide, and prednisone protocol [17]. Retrospective analysis of 283 myeloma patients showed that inverse PLR had predictive value for OS and PFS [18].

Despite recent interest in the clinical implications of activated platelets in the setting of cancer, the scope of available data is still limited by the type of malignancy, sample sizes, selected population and clinical outcomes studied. PDW is a measure of platelet heterogeneity caused by heterogeneous demarcation of megakaryocytes. Several cytokines such as IL6, granulocytes colony stimulating factor (G-CSF) and macrophage colony stimulating factor (M-CSF) have dual functions including regulating megakaryopoiesis and tumour progression [19]. Another possible mechanism is that activated platelets create a procoagulant micro-environment that protect the tumour cells from the host immune system [20].

Increased PDW was found in gastric cancer and lung cancer [21, 22], and has been demonstrated to have a poor prognostic impact in melanoma, thyroid cancer, colorectal cancer, and laryngeal cancer. Also, studies, found that an increased PDW was associated with advanced TNM stages and shortened OS in patients with nasopharyngeal cancer. In contrast, other studies showed that decreased PDW was found in thyroid and breast cancer [23,24], and is an unfavourable predictive factor for non-small cell lung cancer patient survival [25].

To the best of our knowledge, our study is the first to demonstrate the effect of high PDW in CLL patients and it revealed that High PDW ( $\geq 18.5 \mathrm{fl}$ ) was more frequently associated with intention to treat population $(p=0.038)$, and CD38 expression ( $p=0.068)$, but not with $17 p$ deletion $(p=0.25)$ and seropositive HCV patients $(p=0.4)$.

\section{Conclusions}

The low PLR and high PDW are associated with poor prognostic markers in CLL patients. CLL staging and PDW are independent predictors of survival. Unfortunately, the other prognostic markers as 17p deletion, CD38 and ZAP-70 were not performed for all our patients. We recommend further prospective studies to evaluate these simple applicable and cheap biomarkers in larger numbers of patients.

Conflict of interest: none declared

\section{Shaimaa El-Ashwah \\ Mansoura University \\ Oncology Center \\ 35516 Mansoura, Egypt \\ e-mail:shaimaelashwah@gmail.com}

Received: 14 Mar 2020

Accepted: 26 Apr 2020

\section{References}

1. Bannerji R, Byrd JC. Update on the biology of chronic lymphocytic leukemia. Curr Opin Oncol. 2000; 12(1): 22-29, doi: 10.1097/00001622200001000-00004, indexed in Pubmed: 10687725.

2. Chiorazzi N, Rai KR, Ferrarini M. Chronic lymphocytic leukemia. N Engl J Med. 2005; 352(8): 804-815, doi: 10.1056/NEJMra041720, indexed in Pubmed: 15728813.

3. Burger J, Tsukada N, Burger M, et al. Blood-derived nurse-like cells protect chronic lymphocytic leukemia B cells from spontaneous apoptosis through stromal cell-derived factor-1. Blood. 2000; 96(8): 2655-2663, doi: 10.1182/blood.v96.8.2655.

4. Tsang M, Parikh SA. A Concise Review of Autoimmune Cytopenias in Chronic Lymphocytic Leukemia. Curr Hematol Malig Rep. 2017; 12(1): 29-38, doi: 10.1007/s11899-017-0366-1, indexed in Pubmed: 28197963.

5. Dührsen $U$, Augener W, Zwingers $T$, et al. Spectrum and frequency of autoimmune derangements in lymphoproliferative disorders: analysis of 637 cases and comparison with myeloproliferative diseases*. British Journal of Haematology. 2008; 67(2): 235-239, doi: 10.1111/j.1365$-2141.1987 .00235 . x$.

6. Hallek M, Cheson BD, Catovsky D, et al. International Workshop on Chronic Lymphocytic Leukemia. Guidelines for the diagnosis and treatment of chronic lymphocytic leukemia: a report from the International Workshop on Chronic Lymphocytic Leukemia updating the National Cancer Institute-Working Group 1996 guidelines. Blood. 2008; 111(12): 5446-5456, doi: 10.1182/blood-2007-06-093906, indexed in Pubmed: 18216293.

7. Rai KR, Sawitsky A, Cronkite EP, et al. Clinical staging of chronic lymphocytic leukemia. Blood. 1975; 46(2): 219-234, doi: 10.1182/blood. v46.2.219.bloodjournal462219.

8. Binet JL, Leporrier M, Dighiero G, et al. A clinical staging system for chronic lymphocytic leukemia.Prognostic significance. Cancer. 1977; 40(2): 855-864, doi: 10.1002/1097-0142(197708)40:2<855::aid-cncr2820400239>3.0.co;2-1.

9. Amaya-Chanaga $\mathrm{Cl}$, Rassenti LZ. Biomarkers in chronic lymphocytic leukemia: Clinical applications and prognostic markers. Best Pract Res Clin Haematol. 2016; 29(1): 79-89, doi: 10.1016/j.beha.2016.08.005, indexed in Pubmed: 27742074.

10. Wang S, Ma Y, Sun L, et al. Prognostic Significance of Pretreatment Neutrophil/Lymphocyte Ratio and Platelet/Lymphocyte Ratio in Patients with Diffuse Large B-Cell Lymphoma. Biomed Res Int. 2018; 2018 : 9651254, doi: 10.1155/2018/9651254, indexed in Pubmed: 30643825.

11. Molica S, Alberti A. Prognostic value of the lymphocyte doubling time in chronic lymphocytic leukemia. Cancer. 1987; 60(11): 2712-2716, doi: 10.1002/1097-0142(19871201)60:11<2712::aid-cncr2820601122>3.0.co;2-1.

12. Koller C, Bekele BN, Zhou X, et al. Plasma thrombopoietin compared with immunoglobulin heavy-chain mutation status as a predictor of survival in chronic lymphocytic leukemia. Blood. 2006; 108(3): 1001-1006, doi: 10.1182/blood-2005-05-2110, indexed in Pubmed: 16551975. 
13. Yu H, Xu W, Shen QD. Serum levels of soluble CD (23) and thrombopoietin in chronic lymphocytic leukemia. Zhonghua nei ke za zhi. 2008; 47(10): 826-829.

14. Zent CS, Kay NE. Autoimmune complications in chronic lymphocytic leukaemia (CLL). Best Pract Res Clin Haematol. 2010; 23(1): 47-59, doi: 10.1016/j.beha.2010.01.004, indexed in Pubmed: 20620970.

15. Wang $\mathrm{Y}$, Attar BM, Fuentes HE, et al. Evaluation of the prognostic value of platelet to lymphocyte ratio in patients with hepatocellular carcinoma. J Gastrointest Oncol. 2017; 8(6): 1065-1071, doi: 10.21037/ jgo.2017.09.06, indexed in Pubmed: 29299368

16. Kang HJ, Kim WS, Kim SJ, et al. Phase II trial of rituximab plus CVP combination chemotherapy for advanced stage marginal zone lymphoma as a first-line therapy: Consortium for Improving Survival of Lymphoma (CISL) study. Ann Hematol. 2012; 91(4): 543-551, doi: 10.1007/s00277011-1337-6, indexed in Pubmed: 21922208.

17. Seo J, Kim WS, Kim JS, et al. Platelet to lymphocyte ratio (PLR) retains independent prognostic significance in advanced stage marginal zone lymphoma patients treated with rituximab, cyclophosphamide, vincristine, and prednisone combination chemotherapy (R-CVP): Consortium for Improving Survival of Lymphoma trial. Blood Res. 2017; 52(3): 200-206, doi: 10.5045/br.2017.52.3.200, indexed in Pubmed: 29043235.

18. Jung SH, Kim JS, Lee WS, et al. Prognostic value of the inverse platelet to lymphocyte ratio (iPLR) in patients with multiple myeloma who were treated up front with a novel agent-containing regimen. Ann Hematol. 2016; 95(1): 55-61, doi: 10.1007/s00277-015-2521-x, indexed in Pubmed: 26467916.
19. Zhang $\mathrm{H}$, Liu Li, Fu S, et al. Higher platelet distribution width predicts poor prognosis in laryngeal cancer. Oncotarget. 2017; 8(29): 48138 48144, doi: 10.18632/oncotarget.18306, indexed in Pubmed: 28624815.

20. Santilli F, Boccatonda A, Davì G. Aspirin, platelets, and cancer: The point of view of the internist. Eur J Intern Med. 2016;34: 11-20, doi: 10.1016/j. ejim.2016.06.004, indexed in Pubmed: 27344083.

21. Oncel M, Kiyici A, Oncel M, et al. Evaluation of Platelet Indices in Lung Cancer Patients. Asian Pac J Cancer Prev. 2015; 16(17): 7599-7602, doi: 10.7314/apjcp.2015.16.17.7599, indexed in Pubmed: 26625768.

22. Gunaldi M, Erdem D, Goksu S, et al. Platelet Distribution Width as a Predictor of Metastasis in Gastric Cancer Patients. J Gastrointest Cancer. 2017; 48(4): 341-346, doi: 10.1007/s12029-016-9886-5, indexed in Pubmed: 27826813.

23. Okuturlar Y, Gunaldi M, Tiken EE, et al. Utility of peripheral blood parameters in predicting breast cancer risk. Asian Pac J Cancer Prev. 2015; 16(6): 2409-2412, doi: 10.7314/apjcp.2015.16.6.2409, indexed in Pubmed: 25824773.

24. Yaylaci S, Tosun O, Sahin O, et al. Lack of Variation in Inflammatory Hematological Parameters between Benign Nodular Goiter and Papillary Thyroid Cancer. Asian Pac J Cancer Prev. 2016; 17(4): 2321-2323, doi: 10.7314/apjcp.2016.17.4.2321, indexed in Pubmed: 27221938.

25. Cui MM, Li Na, Liu X, et al. Platelet distribution width correlates with prognosis of non-small cell lung cancer. Sci Rep. 2017; 7(1): 3456, doi: 10.1038/s41598-017-03772-z, indexed in Pubmed: 28615714. 